Edward G. Schmidt

Department of Physics and Astronomy

University of Nebraska

Lincoln, Nebraska, U.S.A.

Abstract. A program is underway to obtain accurate, well sampled light curves for Cepheids in several Local Group galaxies. We have succeeded in attaining accuracies adequate for light curve analysis for stars with periods as short as about 12 days. Photometry for six known Cepheids in NGC 6822 is discussed. Although better sampling in phase is needed to allow full light curve analysis, a Fourier decomposition was performed for one star and it appears to show some differences from galactic Cepheids. A preliminary comparison of some of the gross properties of the light curves with galactic Cepheids shows both similarities and interesting differences.

\title{
PAST STUDIES
}

The study of Local Group Cepheids has a long history going back at least three quarters of a century to the discovery of the period-luminosity relation in the Magellanic Clouds by Leavitt (1912). Since that time the relation has been basic both to the application of Cepheids to the determination of the cosmic distance scale and to studies of the properties of the stars themselves.

A decade and a half later, Hubble applied the period-luminosity relation to show that some objects which were then referred to as "nebulae" were in fact outside of the Milky Way and were galaxies in their own right. The first such "extragalactic nebulae" was NGC 6822 (Hubble 1925). We have obtained new photometric data for Cepheids in this dwarf irregular galaxy and will discuss them below.

Since the time of Hubble, the Cepheids have continued to play a central role in the determination of the distance scale within the Local Group. Some recent workers have recalibrated the older photographic photometry using modern techniques (for example, Sandage 1983 and Christian \& Schommer 1987 for M 33; Freedman 1988 for IC 1613). Others have obtained new data in longer wavelength bands such as in $R$ and $I$ (for example, Freedman, Grieve \& Madore 1985; Walker 1987; Freedman, 1988; Hodge, Lee \& Mateo 1988) or in the near infrared (for example Madore et al. 1985). The emphasis has been on obtaining a few points which can be used in distance determination; of the cited authors, only walker has attempted to obtain relatively complete light curves. 
It is obvious from even this brief survey of the literature that most of the work on extragalactic Cepheids has been concerned with their use in distance determination while much less attention has been given to the study of the stars' own properties. No doubt this is due in part to the great importance of the distance scale in many areas of astronomy as well as the great difficulties it presents. The distance scale has both deserved and required all the attention it has received.

The faintness of extragalactic Cepheids has also been an impediment to their study. Except for the Magellanic Cloud Cepheids, only broad band light and sometimes color curves are available and they are not of good accuracy. This has limited most studies to the consideration of statistical properties derived from the light curves. For example Becker, Iben \& Tuggle (1977) compared the period-frequency relations in several galaxies in an attempt to infer the star formation histories. However, selection effects can seriously distort period-frequency relations and other statistical properties of the Cepheid population.

A persual of photographic light curves for these stars (see for example Kayser 1961 for NGC 6822 and Sandage 1983 and Sandage \& Carlson 1983 for $M$ 33) shows that the scatter is several tenths of a magnitude at best and is often worse. This scatter differs from star to star and frequently light curves bottom out due to background effects. While this data is mostly adequate for the purpose for which it was collected, providing mean magnitudes and periods for distance determination, clearly the form of the light curves is lost in the noise.

A further difficulty in the study of light curves has been the lack of a firm theoretical basis for their interpretation. Hydrodynamic codes have not been able to reproduce observed light curves sufficiently well to restrict the parameters of the models (see Simon 1988). Additionally, comparisons of light curves have often been very subjective in nature.

\section{CURRENT PROSPECTS}

The situation described above is now changing. The theoretical interpretation of light curves is on firmer ground due to the development of Fourier decomposition while new detectors have greatly improved photometry at faint levels.

In the method of Fourier decomposition, the light curve of a variable star is fitted with a Fourier series. The amplitudes and phases of the various terms are combined to yield parameters which allow a quantitative comparison of light curves. This technique has been applied to a variety of stars including classical Cepheids, type II Cepheids, RR Lyrae stars in globular clusters and RR Lyrae stars in.the field (see Simon 1988 for a review).

Simon \& Moffett (1985) have presented Fourier decompositions for the classical Cepheids. In their plots of the Fourier parameters against period the changing asymmetry of the light curves and the presence of 
the period resonance near ten days are clearly seen. Overtone pulsators are also discriminated in those plots. Similar studies have been conducted for Cepheids in the Large and Small Magellanic Clouds by Andreasen \& Peterson (1987) and Andreasen (1988a). Although the scatter in the diagrams is larger, it is possible to distinguish differences between those Cepheids and the galactic Cepheids. In particular the resonance at ten days appears at slightly longer periods among the Magellanic Cloud Cepheids.

The use of Fourier decomposition has partially although not totally alleviated the difficulties with light curve interpretation. The subjective nature of the comparisons have been removed while the appearance of the period resonance gives us information on the interiors of the stars. However, we are not yet able to reproduce the sequences of Fourier coefficients from hydrodynamic models. This should be an important goal for future theoretical studies.

Although, the Fourier technique has proved to be a great benefit to studies of light curves, it must be remembered that it is very demanding of the data. Good photometric accuracy is necessary to obtain reliable Fourier coefficients. Uniform phase coverage is required because gaps in the light curves result in unstable coefficients.

The introduction of solid state panoramic detectors, the CCD in particular, has opened the way to obtaining much better photometric data at faint levels than was possible with older techniques. These detectors offer better photometric properties than the photographic plate (for example, higher quantum efficiency, large dynamic range, linearity, good stability) while still being panoramic. This latter point is important in crowded fields where overlapping star images must be deconvolved.

Figure 1. Calculated accuracy of CCD photometry.

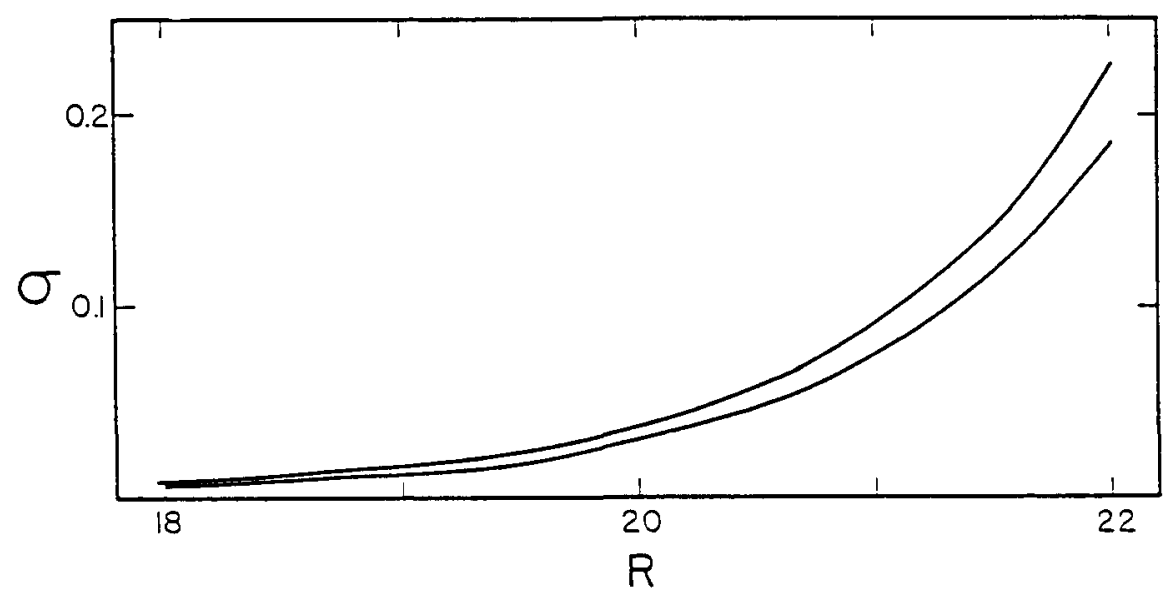


The good phase coverage required by the Fourier decomposition technique dictates the use of a small telescope. The quantum efficiency of the CCD makes it possible to obtain the needed accuracy in exposure times which are not prohibitive. In Figure 1 the photometric error in the Cousins $R$ band due to photon statistics in the star signal and the background is plotted against the brightness of the star in the $R$ band. This calculation assumes an 0.8-meter telescope and an exposure of 45 minutes. The background is an important contributor to the error and two values were used. The upper curve is with the measured background at Kitt Peak National observatory while the lower is for the background brightness measured at McDonald Observatory. In each case, a contribution from the galaxy is included. This latter naturally varies from one galaxy to another and within a galaxy. We have used a brightness which is from our field \#1 in NGC 6822. A seeing of two arc seconds (FWHM) has been assumed. It can be seen that with these assumptions, which are not particularly demanding for a good site, it is possible to obtain errors of a tenth of a magnitude at $R=21$. This corresponds to minimum light for Cepheids with periods of about six days in NGC 6822 and nine days in M 33. Thus, a small telescope at a dark site is capable of obtaining data of sufficient quality to study light curves for extragalactic Cepheids.

\section{OUR PROGRAM}

Based on the considerations outlined above, we undertook a program aimed at obtaining accurate, well-sampled light curves for Cepheids in several Local Group galaxies. Observations have been made with CCD cameras on the 0.8-meter telescope at McDonald observatory and the 0.9-meter and 2.1-meter telescopes at Kitt Peak National observatory. We need to obtain data on enough stars in each system to allow us to learn the overall properties of the Cepheids but must minimize the number of fields observed in each to keep the program within feasible limits. The results of this program will be of more general use if they are reduced to a standard photometric system even though that is not necessary to realize the primary goals.

In order to carry out Fourier decomposition of a light curve, a minimum of about 30 well spaced data points are needed. In our fields there are a mixture of Cepheids of different periods. Thus, it is not possible to optimize the observing times to get even spacing in phase for more than a few of them. This, together with telescope scheduling, dictates that we must obtain enough data points to fill in the light curves in a random fashion. About 45 frames in each field will accomplish this for the bulk of the stars.

In Table 1 we list the galaxies for which we have obtained data. The two fields in each were selected to maximize the number of known Cepheids within the three by five arc minute area of the CCD. It can be seen from the table that we will be able to obtain data for between eight and forty Cepheids in each galaxy. It will also be noted that we have obtained nearly enough frames to meet our needs in three of the four galaxies on the list. 
We have chosen to obtain our data through filters matched to the Cousins $R$ and $I$ bands. $R$ will constitute the primary color for use in the Fourier decomposition and fewer I exposures will be obtained for the purpose of providing colors. The reliability of Fourier decompositions is dependent on the size of the photometric errors compared with the star's amplitude. Although the amplitude in the $\mathrm{R}$ band is $2 / 3$ that in the $V$ band, the greater sensitivity of the $C C D$ at longer wavelengths allows us to obtain smaller relative errors in shorter times. In fact, with some of the CCD's we have used in this project, the exposures in $V$ would have been prohibitive. The choice of I to provide a color was dictated by the fact that the amplitude in that band is sufficiently small to allow us to interpolate in the I light curve and form colors even if it is relatively poorly sampled.

Our experience with Fourier decomposition of light curves indicates that we need accuracy of several hundredths of a magnitude to carry out such an analysis. Based on the above discussion, we can expect to achieve this accuracy for Cepheids with periods longer than about twelve days in $R$ with exposures of about an hour while the exposures in I will generally be less. Longer exposures will be used in some of the fields to extend our sample to shorter periods.

We will reduce the photometry to the $R$ and I system of Cousins. With the CCD and suitable filters it has been possible to achieve accurate transformations to that system (Walker 1984; Schmidt 1988).

Although the number of known Cepheids in our fields are sufficient for our needs, clearly the value of this work would be enhanced if we could increase the number. There is reason to believe that, in fact, for $M 33$ and NGC 6822 our fields contain many undiscovered Cepheids.

In NGC 6822 there are thirteen known Cepheids. On the other hand, each of the Magellanic Clouds contains about 1100 known Cepheids. Scaling this by the galaxy masses or following Helfand's (1984) scaling of x-ray

Table 1

Fields in Local Group Galaxies

\begin{tabular}{ccccc} 
Galaxy & $\begin{array}{c}\text { Field } \\
\text { \# }\end{array}$ & \multicolumn{2}{c}{$\begin{array}{c}\text { Number of } \\
\text { frames }\end{array}$} & $\begin{array}{c}\text { Number of } \\
\text { Cepheids }\end{array}$ \\
\hline \multirow{2}{*}{ NGC 6822} & 1 & 40 & 26 & 6 \\
\multirow{2}{*}{ M33 } & 2 & 31 & 16 & 4 \\
& 1 & 39 & 20 & 6 \\
M31 & 2 & 35 & 14 & 2 \\
IC 1613 & 1 & 41 & 25 & 20 \\
& 2 & 27 & 17 & 25 \\
& 1 & 10 & 8 & 10 \\
& 2 & 4 & 2 & 8 \\
\hline
\end{tabular}


binary rates or supernova rates, we find that there should be between 30 and 200 Cepheids in NGC 6822. Our sample could thus be increased by a factor of at least two with a complete search.

An examination of the period-frequency relations of Becker, Iben \& Tuggle (1977) also indicates that there are short period stars missing in the samples for M 33 and NGC 6822. Extrapolations using the relations for M 31 and the Milky Way show that there should be between eight and thirteen additional Cepheids with periods between six and twenty days in our field 1 in NGC 6822 and thirteen to sixteen with periods between nine and twenty days in our field 1 in $M$ 33. Again an increase of more than a factor of two is indicated over the present sample.

Finally we note that a study by Freedman (1988) supports these conclusions. She finds that stars within the instability region of the HR diagram in IC 1613 outnumber the known Cepheids by a factor of about two. Additionally, preliminary searches of some of our data for new Cepheids have revealed many new suspected variables (Schmidt \& Spear 1988a).

Based on these considerations, it appears that the number of Cepheids in our sample will be at least twice that listed in Table 1 for $M 33$ and NGC 6822 .

\section{FIRST RESULTS}

We have used the DAOPHOT image reduction package (described by Stetson 1987) to extract magnitudes from part of our data set. This package is useful in crowded fields.

DOAPHOT forms groups of stars with overlapping images which are then fit jointly to a point spread function. In the fields we are studying the groups generally grow to include most of the stars on the frame and are too large to be handled by DAOPHOT. It is obvious that any given program star is not greatly affected by stars some distance away even if there is an overlapping chain of stars between. We have thus limited the groups to several image diameters and fitted each program star in a group with between a half dozen and several dozen stars.

A serious source of error in photometry at faint levels is the presence of background stars. In the case of variable star observations we have a distinct advantage because we are analyzing many exposures of the same field. Additionally, we obtained some exposures with the 2.1-meter telescope at Kitt Peak which offered a larger image scale and thus a better definition of the background around the program stars. Following a first pass in which we allowed DAOPHOT to identify neighboring stars and obtain preliminary magnitudes, we formed a collated list of stars around each program object. We then used this as input to a second pass in which DAOPHOT fit the background with the same set of stars for each frame. The scatter from frame to frame was reduced by about half in the second pass. 
A third difficulty was the unavailability of standard stars in our field. We have overcome this by selecting a number of local comparison stars and have defined instrumental magnitudes for them from a selection of the best frames. In NGC 6822 we have made a preliminary calibration of these stars using the photometry of Hoessel \& Anderson (1986) but will obtain a new calibration using the $\mathrm{CCD}$ at Behlen Observatory. In the meantime, the data discussed here are reduced to the $r$ magnitude in the Thuann and Gunn system.

To determine how close we have come to the expected accuracy discussed above and to determine whether the final accuracy of the extracted magnitudes is adequate to our purposes we have compared the photometry from 32 frames. Since all were accurately reduced to the same system through the use of the local comparison stars, the internal scatter among the various frames will give us an indication of the random errors. The results are plotted against the $r$ magnitude in Figure 2 . The solid circles indicate the standard deviations estimated from the local comparison star magnitudes while the open circles and $x^{\prime} s$ are the errors from other stars which happened to be in the groups with program stars. The + signs indicate the rms scatter in Cepheid light curves during intervals when the star appeared to have little variation. The solid curve is from Figure 1 shifted to correspond to the Thuann and Gunn $r$ magnitude. It can be seen that the actual errors are only about $50 \%$ higher than those estimated from photon statistics alone. This is not surprising in view of the need to fit the backgounds and star images. Methods are being investigated to further reduce the internal errors.

In our field 1 in NGC 6822 we have extracted data for nine known variables of which six are Cepheids. In plotting the new data we found that the periods given by Kayser (1961) for these stars generally produce excess scatter. We have therefore adjusted the periods for all except one of the Cepheids. The older periods and the revised values

Figure 2. Errors of CCD photometry. Various symbols show internal errors as discussed in the text and the solid line is from Figure 1.

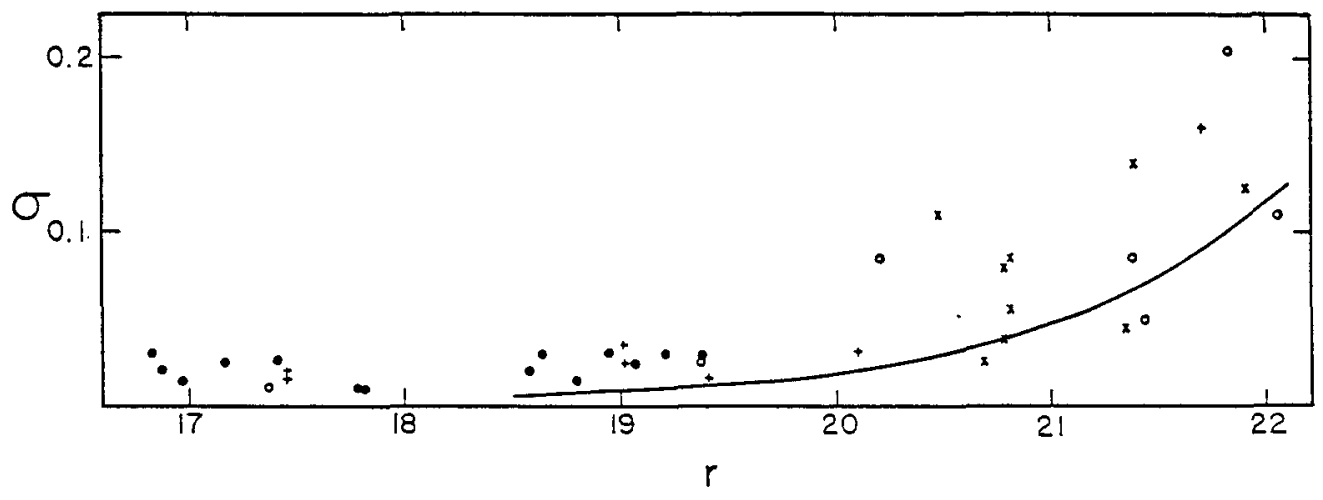


are listed in Table 2. Since the data used by Kayser were obtained over nearly fifty years and there are often gaps of the order of a year in the series, it might be speculated that the erroneous periods result from aliasing with one year. In the last column of Table 2 we list the periods obtained under this assumption; surprisingly only one of the periods, that for $V 6$, can be explained in this way. Unfortunately, our data is not ideally spaced in time to determine good periods and our revised values should not be considered definitive.

The light curves for the six Cepheids using data from two observing seasons, 1985 and 1986, are shown in Figure 3. A comparison of these light curves with the previous photographic light curves (Kayser 1961) shows that we have achieved a scatter which is smaller by two to six times. The shapes of the light curves are thus well defined.

It will be noticed that in several of the light curves there are points which scatter by an abnormally large amount compared with the rest. For example in the light curve of $v 4$ there are three points just after maximum light which scatter by between a tenth and two tenths of a magnitude from the remainder while in $\mathrm{V} 21$ there are two points which fall below the minimum defined by other points by a similar amount. Problems of this type are common in CCD data and reflect undiagnosed cosmic ray hits or defective pixels. Unfortunately, the statistical tests applied by DAOPHOT to the fitting do not seem to effectively discriminate against these occurences. Other means of eliminating or correcting such data points are being investigated but in the mean time we can ignore the few obviously discrepant points.

Although the phase coverage in our light curves is not yet sufficient to carry out a definitive Fourier decomposition, it appeared worthwhile to attempt a very preliminary analysis for two stars, $V 4$ (with three discrepant points omitted) and $V 6$. The parameters derived from the fits are displayed in Table 3 . For each star we give the Fourier parameters for fits of order one through five. It will be noted that the fit for $V 4$ is stable in the sense that the Fourier parameters change very little from one order to the next. On the other hand the

Table 2

Periods of the NGC 6822 Variables

\begin{tabular}{llll} 
Star & $\begin{array}{c}\text { Period } \\
\text { (Kayser) }\end{array}$ & $\begin{array}{c}\text { Period } \\
\text { (revised) }\end{array}$ & $\begin{array}{c}\text { Alias with } \\
\text { one year }\end{array}$ \\
\hline V 5 & 13.3550 & 13.412 & $12.94,13.94$ \\
V 4 & 17.3471 & 17.3471 & $16.56,18.21$ \\
V 21 & 17.457 & 17.51 & $16.71,18.39$ \\
V 6 & $21.1450 *$ & 19.986 & $18.95,21.14$ \\
V 28 & 34.6672 & 34.625 & $31.63,38.25$ \\
V 7 & 65.45 & 66.13 & $55.99,80.75$
\end{tabular}

* Alias with one year 
parameters for $V 6$ can be seen to change appreciably and the fitting is clearly unstable. This is no doubt due to the gap in phase coverage near and after maximum light. We can not conclude anything from the Fourier fit to this star.

In Figure 4 the fitted curve for V 4 is plotted with the original data. It can be seen that the fit represents the data well; the scatter about the line is \pm 0.033 magnitudes. Some of the parameters formed from the Fourier coefficients are plotted in Figure 5 on the diagrams for classical Cepheids (from Simon \& Moffett 1985). For $R_{21}$ and $\Phi_{21}, V_{4}$ matches the classical Cepheids well. However, the discrepancy for $\Phi_{31}$ is too large to attribute to uncertainties in the fit and may indicate differences between the classical Cepheids and the Cepheids in NGC 6822 .

Figure 3. Light curves for Cepheids in NGC 6822.
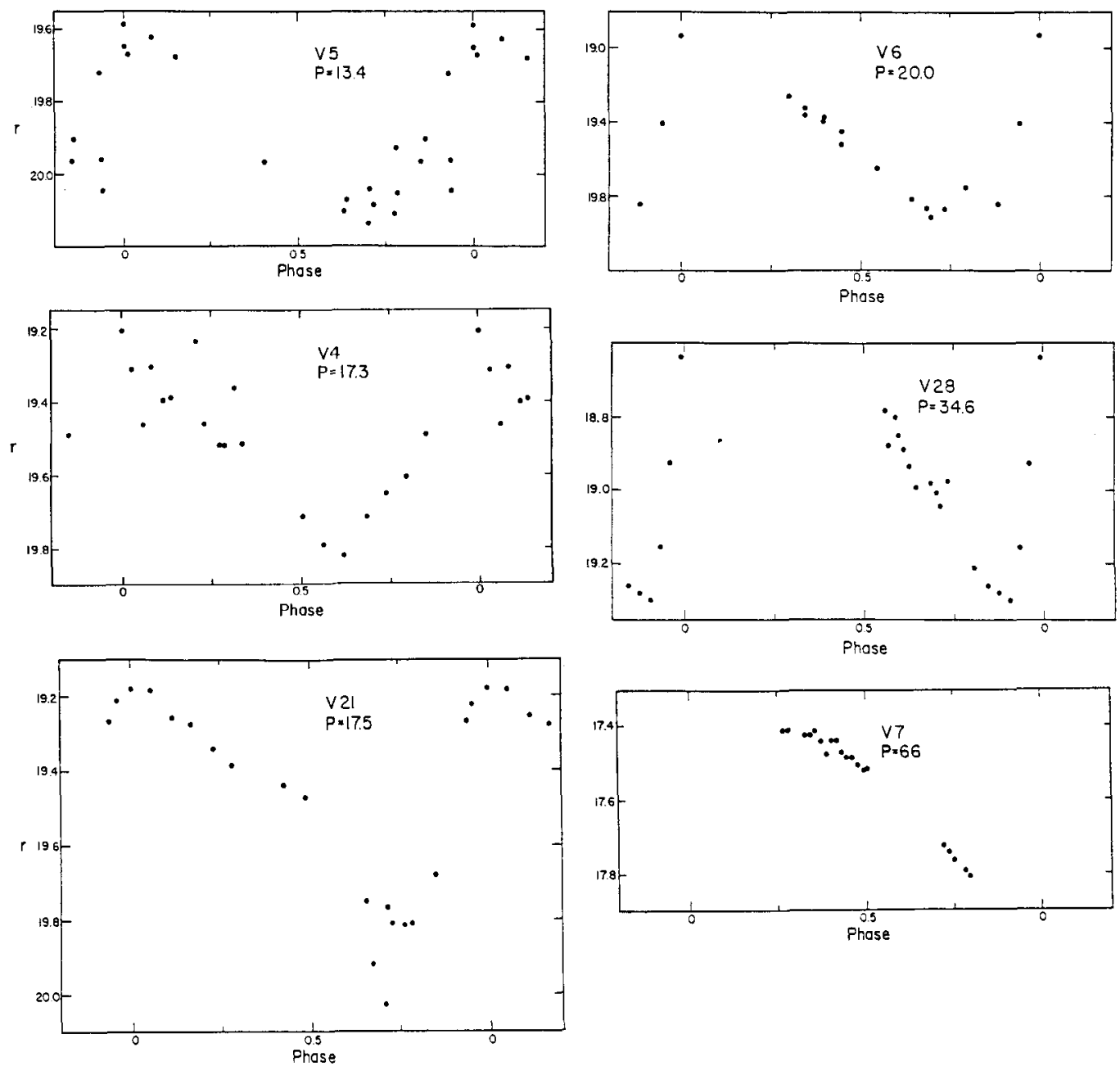
Fourier decompositions of more stars are needed to determine the significance of this tantalizing result.

Although there is not yet enough data to carry out Fourier decompositions, we can make some comparisons of gross light curve

Table 3

Fourier Fits for Variable V 4

\begin{tabular}{cccccc} 
Order & $\begin{array}{c}\text { Std.Dev. } \\
(0.01 \text { mag) }\end{array}$ & $\begin{array}{c}\mathrm{R}_{21} \\
(\mathrm{mag})\end{array}$ & $\begin{array}{c}\Phi_{21} \\
(\mathrm{rad})\end{array}$ & $\begin{array}{c}\mathrm{R}_{31} \\
(\mathrm{mag})\end{array}$ & $\begin{array}{c}\Phi_{31} \\
(\mathrm{rad})\end{array}$ \\
\hline 1 & 5.3 & & & & \\
2 & 4.1 & 0.26 & 4.76 & & \\
3 & 3.6 & 0.28 & 4.79 & 0.13 & 0.76 \\
4 & 3.0 & 0.34 & 4.84 & 0.12 & 0.75 \\
5 & 3.3 & 0.32 & 4.76 & 0.11 & 1.15
\end{tabular}

Fourier Fits for Variable V 6

\begin{tabular}{cccccc} 
Order & $\begin{array}{c}\text { Std.Dev. } \\
(0.01 \mathrm{mag})\end{array}$ & $\begin{array}{c}\mathrm{R}_{\mathbf{2 1}} \\
(\mathrm{mag})\end{array}$ & $\begin{array}{c}\Phi_{21} \\
(\mathrm{rad})\end{array}$ & $\begin{array}{c}\mathrm{R}_{\mathbf{3 1}} \\
(\mathrm{mag})\end{array}$ & $\begin{array}{c}\Phi_{31} \\
(\mathrm{rad})\end{array}$ \\
\hline 1 & 13.0 & & & & \\
2 & 9.6 & 0.33 & 4.27 & & \\
3 & 5.5 & 0.42 & 3.83 & 0.21 & 1.03 \\
4 & 4.2 & 0.44 & 4.12 & 0.27 & 1.44 \\
5 & 3.0 & 0.38 & 5.21 & 0.37 & 2.98
\end{tabular}

Figure 4. Light curve of V4 with the Fourier fitted curve.

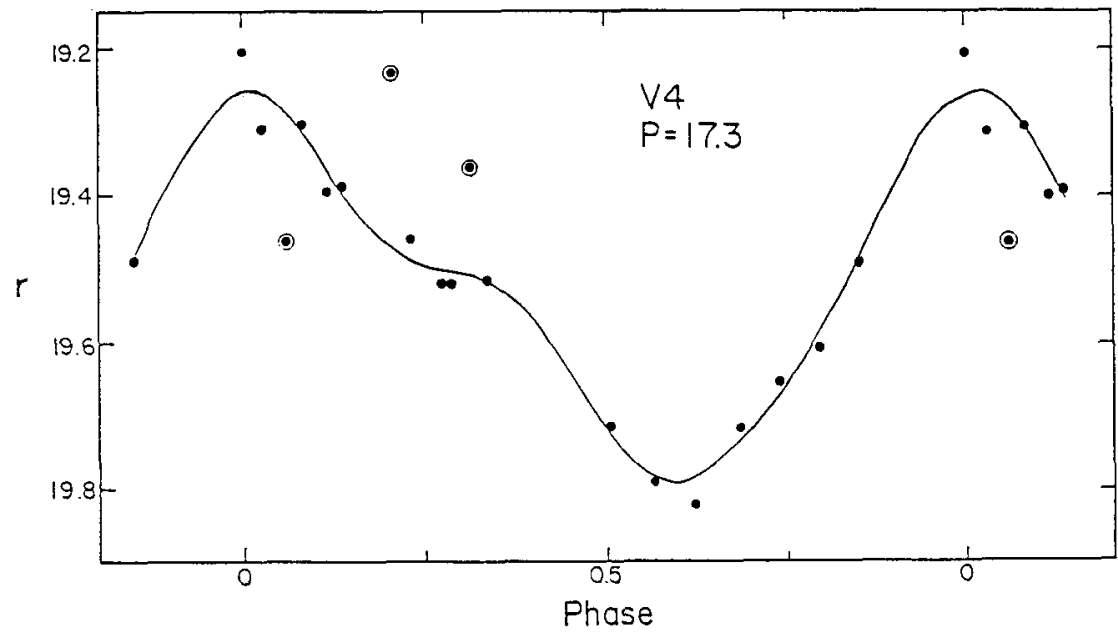


properties between the Milky Way and NGC 6822. Figure 6 shows several of these. There are both similarities and differences between the galactic and the NGC 6822 Cepheids. For example V 21 has a light curve which has a greater skewness and smaller acuteness than any galactic Cepheid of similar period while the rest match the galactic stars quite well. This is obvious from the form of its light curve in Figure 3 . There are also some larger amplitudes and faster decline rates seen in NGC 6822 than for galactic Cepheids.

Figure 5. Fourier diagrams for galactic Cepheids (filled circles) and V4 in NGC 6822 (t signs).
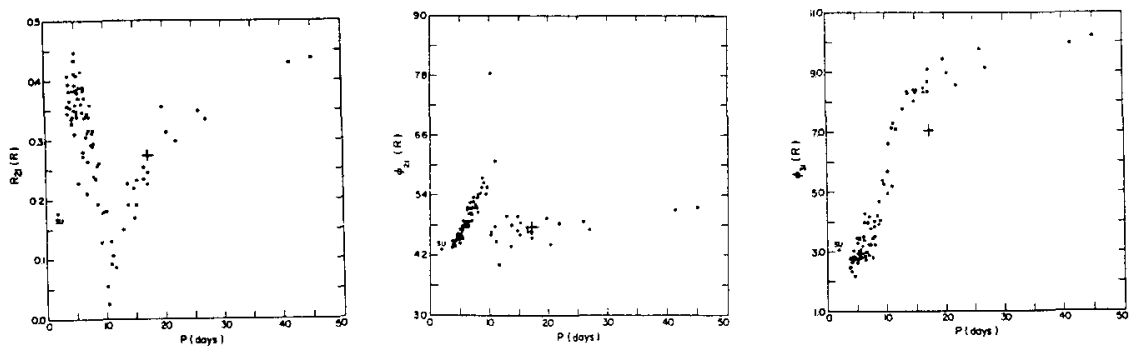

Figure 6. The decline rate, amplitude, skewness and acuteness of galactic Cepheids (filled circles) and Cepheids in NGC 6822 (open circles). For the galactic Cepheids the decline rates were derived from the data of Moffett \& Barnes (1984) while the amplitudes are from Moffett \& Barnes (1985). The skewness and acuteness data for the galactic Cepheids are from Andreasen (1988b).
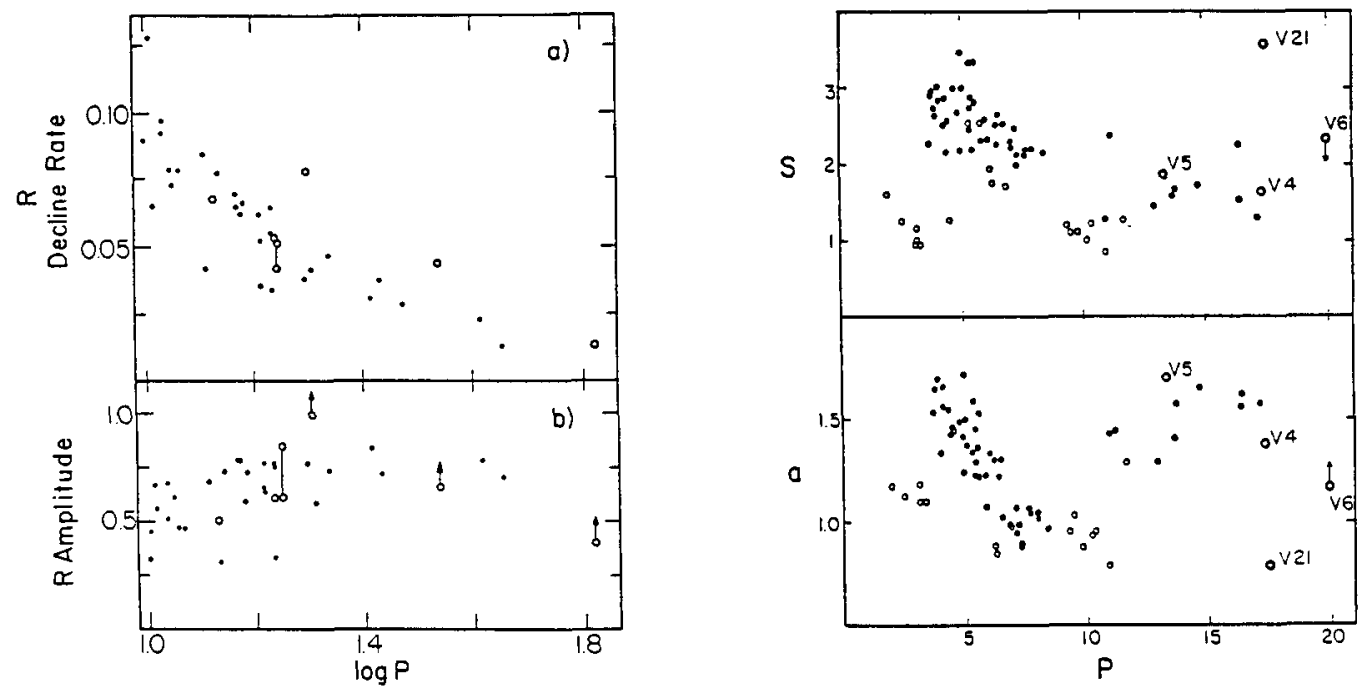
The results so far obtained are not sufficient to attack astrophysical problems. However, it is clear that we are now in a position to utilize the Cepheids in Local Group galaxies as a probe of the stellar content of those galaxies due to the improvements in both interpretation of light curves and in the observational techniques available to obtain them.

\section{ACKNOWLEDGEMENTS}

A number of people have contributed to the program described here and deserve to be thanked. N.R. Simon and T.J. Teays suggested this investigation and provided the impetus to undertake it. G.G. Spear carried out much of the analysis of the data. B.J. Anthony-Twarog developed the image processing facility at the MidAmerica Image Processing Laboratory at the University of Kansas which was used for the reductions. She also provided considerable aid in its use and instructed me in the art of image processing. The observations were carried out at McDonald Observatory of the University of Texas and at Kitt Peak National Observatory. The project was supported by NSF grant AST-8312649.

\section{REFERENCES}

Andreasen, G. K. (1988a). Astron. \& Astrophys.,191, 71. Andreasen, G.K. (1988b). Astron. \& Astrophys.,196, 159.

Andraesen, G.K. \& Petersen, J.O. (1987). Astron. \& Astrophys.,180, 129. Becker, S.A., Iben, I. \& Tuggle, R.S. (1977). Ap.J.,218, 633.

Christian, C.A. \& Schommer, R.A. (1987). A.J., 93, 557.

Freedman, W.L. (1988). Ap.J., 326, 691.

Freedman, W.L., Grieve, G.R. \& Madore B.F. (1985). Ap.J.Suppl.,59, 311. Helfand, D.J. (1984). P.A.S.P., 96, 913.

Hodge, P., Lee, M.G. \& Mateo, M. (1988). Ap.J., 324, 172.

Hoessel, J.G., \& Anderson, N. (1986). Ap.J.SuppI.,60, 507.

Hubble, E. (1925). Ap.J., 62, 409.

Kayser, S.E. (1961). Astron.J., 72, 134.

Leavitt, H.S. (1912). Harvard Círc. no. 173.

Madore, B.F., McAlary, C.W., McLaren, R.A., Welch, D.L., Neugebauer, G., \& Matthews, K. (1985). Ap.J.,294, 560.

Moffett, T.J., Barnes, T.G. (1984). Ap.J.Suppl.,55, 389.

Moffett, T.J., Barnes, T.G. (1985). Ap.J.Suppl., $\overline{58}, 843$.

Sandage, A. (1983). A.J., 88, 1108.

Sandage, A. \& Carlson, G. (1983). Ap.J.,267, L25.

Schmidt, E.G. (1988). In Proceedings of the Ninth Annual

Fairborne-S̄ithsonian-IAPPP Symposium, ed. D. Hayes.

Phoenix: Fairborn Observatory Press.

Schmidt, E.G. \& Spear, G.G. (1988a). B.A.A.S.,19,1036.

Schmidt, E.G. \& Spear, G.G. (1988b). in press M.N.R.A.S.

Simon, N.R. (1988). In Stellar Pulsation and Mass Loss, eds. R. Stalio \& L.A. Wilson, p. 27. Dordrecht: D. Reidel.

Simon, N.R. \& Moffett, T.J. (1985). P.A.S.P.,97, 1078. 
Stetson, P.B. (1987). P.A.S.P.,99, 191.

Walker, A.R. (1984). M.N.R.A.S.,209, 83.

Walker, A.R. (1987). M.N.R.A.S., $\underline{225}, 627$. 